\title{
Comportamento de espécies florestais amazônicas quanto à luminosidade
}

\author{
Jurandyr da Cruz Alencar ( $\left.{ }^{(}\right)$ \\ Vivaldo Campbell de Araujo (**)
}

\begin{abstract}
Resumo
Os autores comparam o crescimento em diâmetro, altura e a sobrevivência de 21 espécies florestais da Amazônia, de interesse econômico, plantadas em duas condiçōes de luminosidade: uma sob sombra de floresta primária nāo explorada e a outra em plena abertura. Verificaram que o crescimento em altura, diâmetro e a porcentagem de sobrevivência, em plena abertura, foi superior ao plantio sob sombra para quase todas as es. pécies testadas, mostrando que a luz é um fator de im. portância fundamental tanto como limitante do crescimento como regulador da sobrevivência de espécies florestais. As espécies que apresentaram melhor comportamento, com maiores alturas e diâmetros em plena abertura, foram Goupia glabra, Cedrelinga catenaeformis, Bagassa guianensis, Carapa guianensis, Jacaranda paraensis, Dipteryx odorata, Calophyllum angulare, Pi. thecolobium racemosum e Scleronema micranthum. Sob sombra destacaram-se Goupia glabra, Jacaranda paraensis, Cedrelinga catenaeformis, Calophyllum angulare, Ocotea rubra e Tabebuia serratifolia. Os autores recomendam estas espécies para plantios na Amazônia, chamando a atenção para o fato de que o conhecimento das características ecológicas intrínsecas de cada espécie é essencial para o êxito da silvicultura nesta regiâo,
\end{abstract}

\section{INTRODUÇÃo}

Este é um trabalho de divulgação dos experimentos silviculturais estabelecidos na Reserva Ducke, e tem por objetivo principal analisar o comportamento de espécies florestais em duas condições de plantio: à sombra de floresta primária não explorada e em plena abertura.

Mesmo com as informações já conhecidas, há ainda falta de dados silviculturais que possibilitem a instalação de plantios em escala industrial, e este trabalho pode trazer uma contribuição neste sentido. Antes de determinarem-se as exigências em nutrientes, espaça- mentos e tratos silviculturais, é imprescindível o conhecimento do comportamento de cada espécie a um determinado ambiente. Sabe-se também que, em face da natureza do ecossistema amazônico, é muito aconselhável pensarse numa silvicultura que cause menos danos à floresta. Por isto, este experimento teve em vista verificar quais as espécies tolerantes à sombra e quais as que necessitam de mais índice de luz para crescer. O conhecimento da aptidão natural de cada espécie é fator fundamental para o desenvolvimento de uma silvicultura mais realista e que leve em consideração a biologia de cada espécie.

\section{MATERIAL E MÉTODO}

O experimento que consta de 21 espécies florestais, cujas madeiras são de interesse econômico nos mercados local, nacional e exterior, foi feito numa área de 1 hectare, localizada na Reserva Ducke; o solo é do tipo latosso. lo amarelo de textura pesada de acordo com Falesi et al. (1969). A área foi dividida em duas partes por meio de uma entrada com $6 \mathrm{~m}$ de largura. No primeiro tratamento, a floresta foi totalmente derrubada; no segundo, foram eliminadas apenas as palmeiras e cipós que compõem o sub-bosque. O plantio foi realizado em linhas, distanciadas $5 \mathrm{~m}$ entre si, com 15 mudas por espécie em cada tratamento, no espaçamento de $5 \mathrm{~m} \times 2,5 \mathrm{~m}$, utilizando-se mudas de altura média entre $30-60 \mathrm{~cm}$, conforme a espécie, em covas de $15 \mathrm{~cm} \times 15 \mathrm{~cm} \times 30 \mathrm{~cm}$. sem qualquer adubação química. As 21 espécies foram plantadas entre junho 1962 até junho 1966, com replantios no ano seguinte (Fig. 2). Em cada tratamento, foram feitas, anualmente, duas limpezas nas linhas de plantio (maio e dezembro), medições das alturas

(*) - Instituto Nacional de Pesquisas da Amazônia, Manaus.

("*) - Curso de Pós-Graduação do Instituto Nacional de Pesquisas da Amazônia (INPA) e Fundação da Universiaade do Amazonas (FUA) - Ecologia, Manaus. 
e diâmetros e levantamento da sobrevivência. Os dados dendrométricos analisados referemse ao periodo 1970 a 1978.

Em face da baixa sobrevivência de Bagassa guianensis, Platymiscium ulei, Clarisia racemosa, Vouacapoua pallidior, Caryocar villosum e Cedrela odorata, com a maioria dos dados em falta em 1978, estas espécies não foram incluídas na análise estatística. Entretanto, aparecem nas Fig. 1a, 1b, 2 e 3, exceto Platymiscium ulei que apresentava $100 \%$ de mortalidade em ambos os tratamentos. Para as 15 espécies restantes, - considerando ainda a existência de dados em falta, que tornariam inviável a análise de variância, - foram tomados, ao acaso, 10 indivíduos de cada espécie em cada um dos tratamentos. Foi feito um teste de Bartlett para cada uma das duas variáveis (altura e diâmetro à altura do peito) para testar a homogeneidade dos dados e um teste de Tukey (nivel $5 \%$ ), para analisar os contrastes entre as médias das variáveis nos tratamentos.

\section{Riesultados}

Nas fig. 1a e 1b, são apresentadas as curvas de crescimento em altura, referentes ao período 1970 a 1978, com exceção de Platymiscium ulei que, já em 1970, apresentava 100\% de mortalidade tanto à sombra da floresta como em plena abertura. Nestas figuras, verifica-se quanto ao tratamento em plena abertura, que se destacaram em altura as espécies Goupia glabra, Cedrelinga catenaeformis, Bagassa guianensis, Carapa guianensis, Jacaranda paraensis, Dipteryx odorata, Calophyllum angulare, Caryocar villosum, Pithecolobium racemosum e Scleronema micranthum; num segundo grupo, com alturas entre 10 e $5 \mathrm{~m}$, destacaram-se Clarisia racemosa, Aniba duckei, Copaifera multijuga, Mezilaurus itauba, Ocotea rubra, Tabebuia serratifolia, Aniba canelilla, Cedrela odorata e Andira parviflora. Vouacapoua pallidior teve igual comportamento como Platymiscim ulei, totalmente mortos já em 1970. O crescimento em altura, sob sombra da floresta. foi inferior ao verificado em plena abertura para todas as espécies testadas, destacandose, entretanto, as espécies Goupia glabra, Ja- caranda paraensis, Cedrelinga catenaeformis, Vouacapoua pallidior, Calophyllum angulare; Ocotea rubra, Tabebuia serratifolia, e Dipteryx odorata; no levantamento feito em 1978, todos os indivíduos de Cedrela odorata, Clarisia racemosa, Bagassa guianensis e a citada Platymiscium ulei estavam totalmente mortos, no tratamento sob sombra, o que mostra serem espécies não tolerantes à sombra e que necessitam de luz para desenvolverem-se.

Quanto à variável D.A.P. (diâmetro à altura do peito), vê-se, pela fig. 2, que as melhores espécies em plena abertura foram Cedrelinga catenaeformis, Carapa guianensis, Jacaranda paraensis, Goupia glabra, Calophyllum angulare, Scleronema micranthum, Dipteryx odorata, Caryocar villosum, Pithecolobium racemosum, Aniba duckei e Mezilaurus itauba. Sob sombra da floresta primária, não submetida à explora. ção comercial, todas as espécies apresentaram também diâmetros médios inferiores aos do plantio em plena abertura. Neste tratamento, as espécies que apresentaram maiores diâmetros foram: Goupia glabra, Jacaranda paraensis, Vouacapoua pallidior, Cedrelinga catenaeformis, Calophyllum angulare, Ocotea rubra, Tabebuia serratifolia, Carapa guianensis. Andira parviflora, Pithecolobium racemosum e Copaifera multijuga apresentaram os menores diâmetros.

A. fig. 3 mostra as porcentagens de sobrevivência anual de cada espécie, onde se verifica que, em 1978, a sobrevivência em plena abertura foi maior do que sob sombra, para todas as espécies testadas, com exceção de Vouacapoua pallidior e Cedrelinga catenaeformis, demonstrando a importância do fator luz, como limitante no desenvolvimento de espécies florestais.

Com referência à análise de 15 espécies, excluindo Platymiscium ulei, Clarisia racemosa, Vouacapoua pallidior, Caryocar villosum e Cedrela odorata que apresentavam, em 1978 alta freqüência de dados em falta, os resultados de um teste de Bartlett revelaram uma heterogeneidade na variância dos dados, tanto para a variável altura como para o diâmetro (D.A.P.). Por isto, uma análise de variância não teria validade. Entretanto, um teste de Tukey ao nível de $5 \%$ de probabilidade, mostrou, para a 

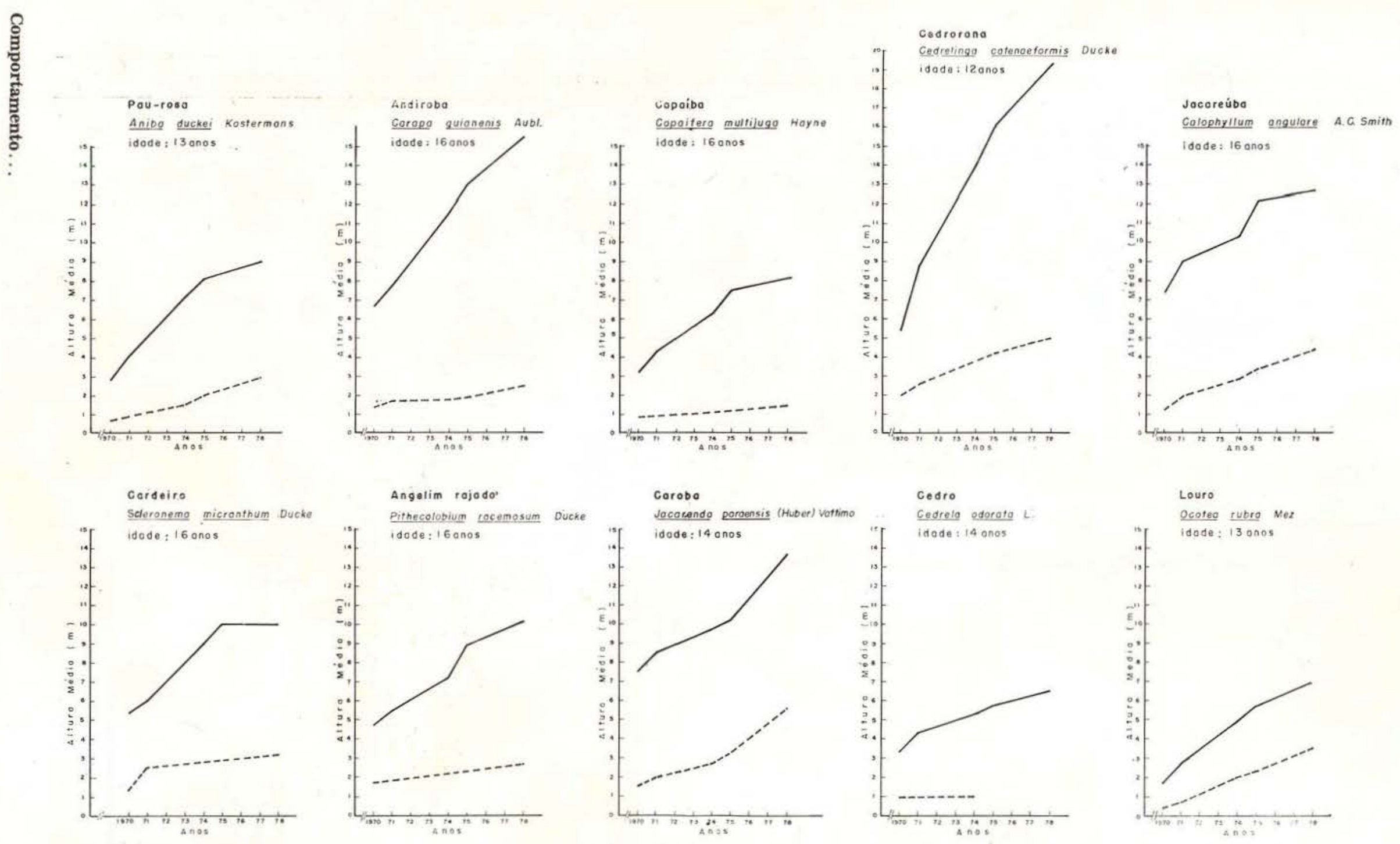

Fig. 1a. - Crescimento em altura $(\mathrm{m})$ de espécies flores tais, plantadas sob sombra de floresta năo explorada (-...) e em plena abertura (-). 


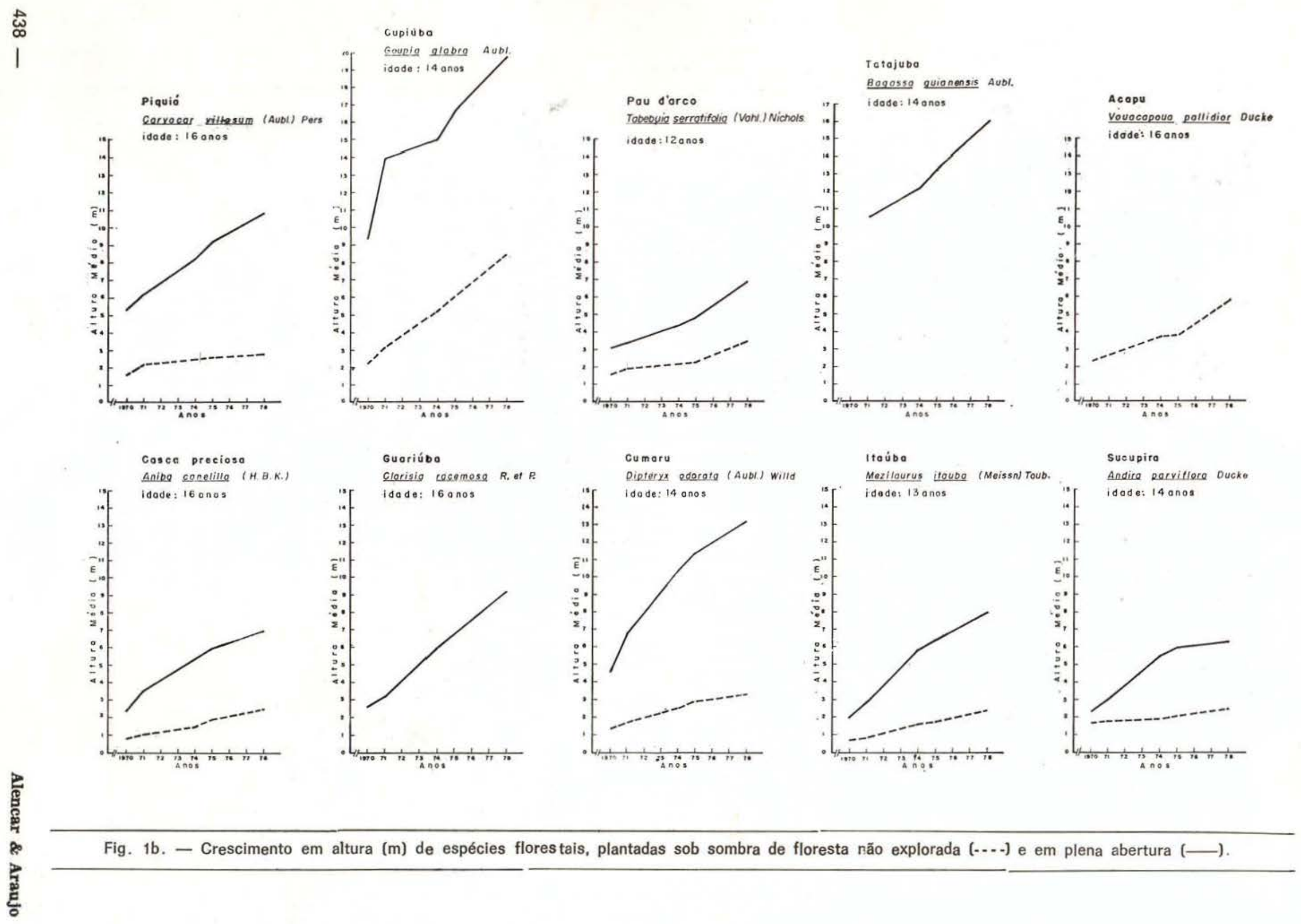




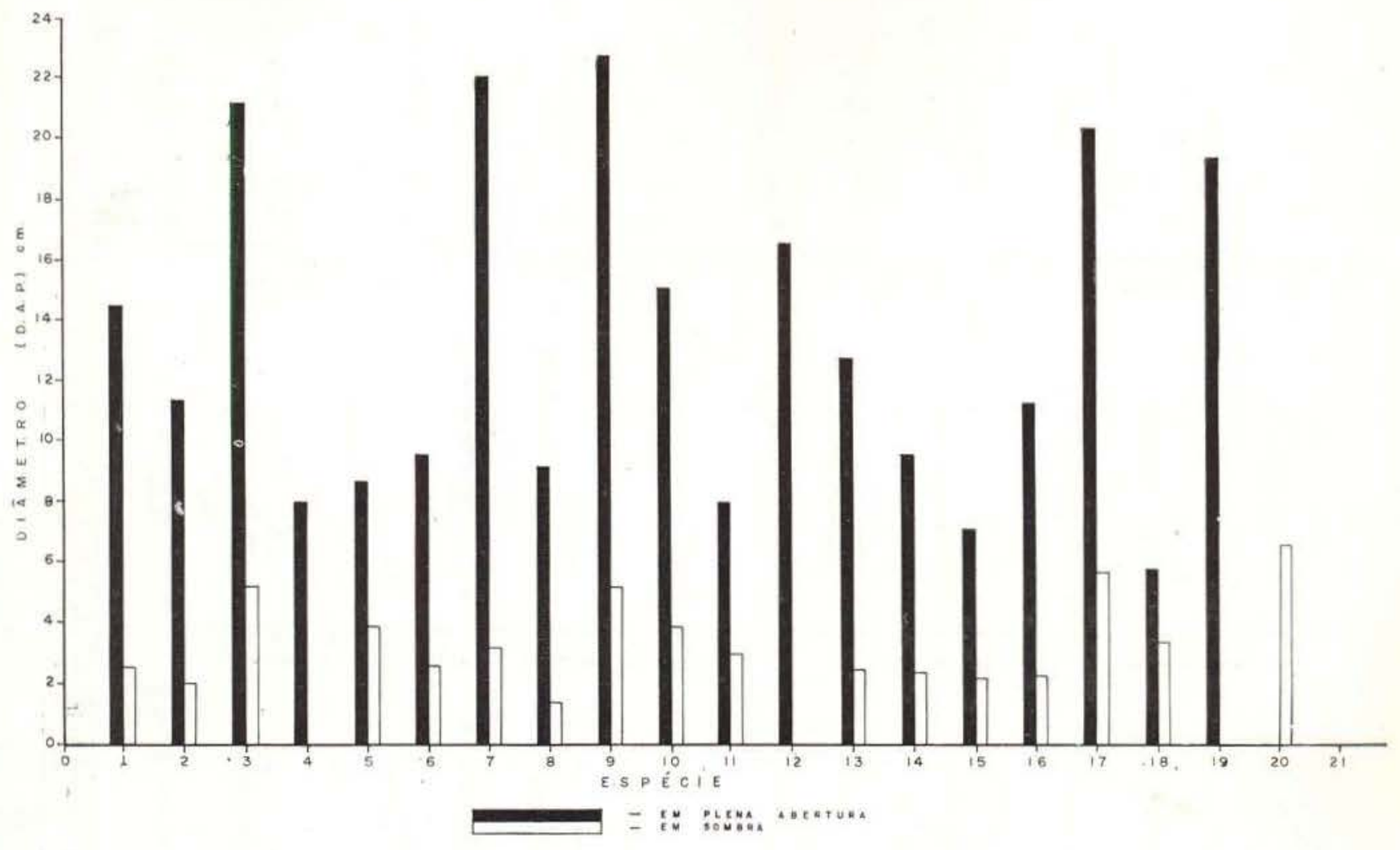

- LE GEN D A -

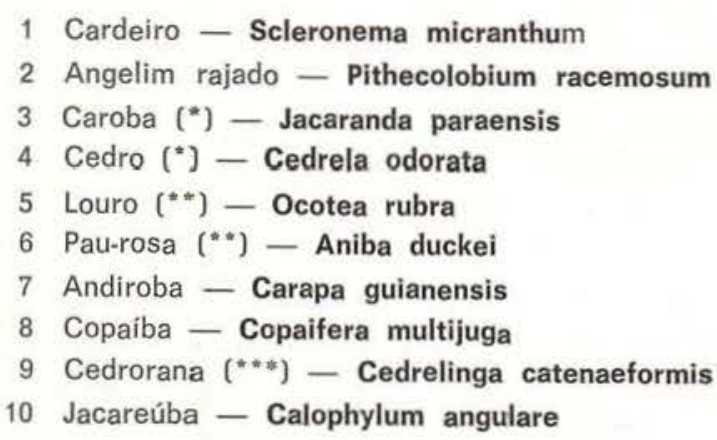

11 Casca preciosa - Aniba canelilla

12 Guariúba - Carisia racemosa

13 Cumaru (*) - Dipteryx odorata

14 Itaúba - Mezilaurus itauba

15 Sucupira $\left({ }^{\circ}\right)$ - Andira parviflora

16 Piquiá - Caryocar villosum

17 Cupiúba (*) - Goupia glabra

18 Pau d'arco (***) - Tabebuia serratifolia

19 Tatajuba (*) - Bagassa guianensis

20 Acapu - Vouacapouo pallidior

21 Macacaúba - Platymiscium ulei

Fig. 2 - Diâmetros médios de plantio sob sombra de floresta primária não explorada e em plena abertura. Idades 16 anos; $14(*) ; 13(* *) ; 12(* *)$.

altura e D.A.P., as médias e o agrupamento das espécies que tiveram igual comportamento (Quadro 1 e 2).

\section{DISCUSSÕES E CONCLUSÕES}

Alguns autores já apresentaram resultados satisfatórios sobre várias espécies florestais como Pedroso et al. (1969), Pedroso (1971), Volpato et al. (1972 e 1973), Alencar \& Fernan- des (1978), Alencar et al. (s/d), mostrando ser possivel hoje definir um grupo de espécies promissoras para a implantação de povoamentos florestais com objetivo industrial.

Sabemos, todavia, pelos ensinamentos da ecologia que a luz, a temperatura e a água representam os fatores ambientais mais relevantes num ecossistema terrestre, não só como limitantes mas também como reguladores. Odum (1977) expõe claramente que nenhum 


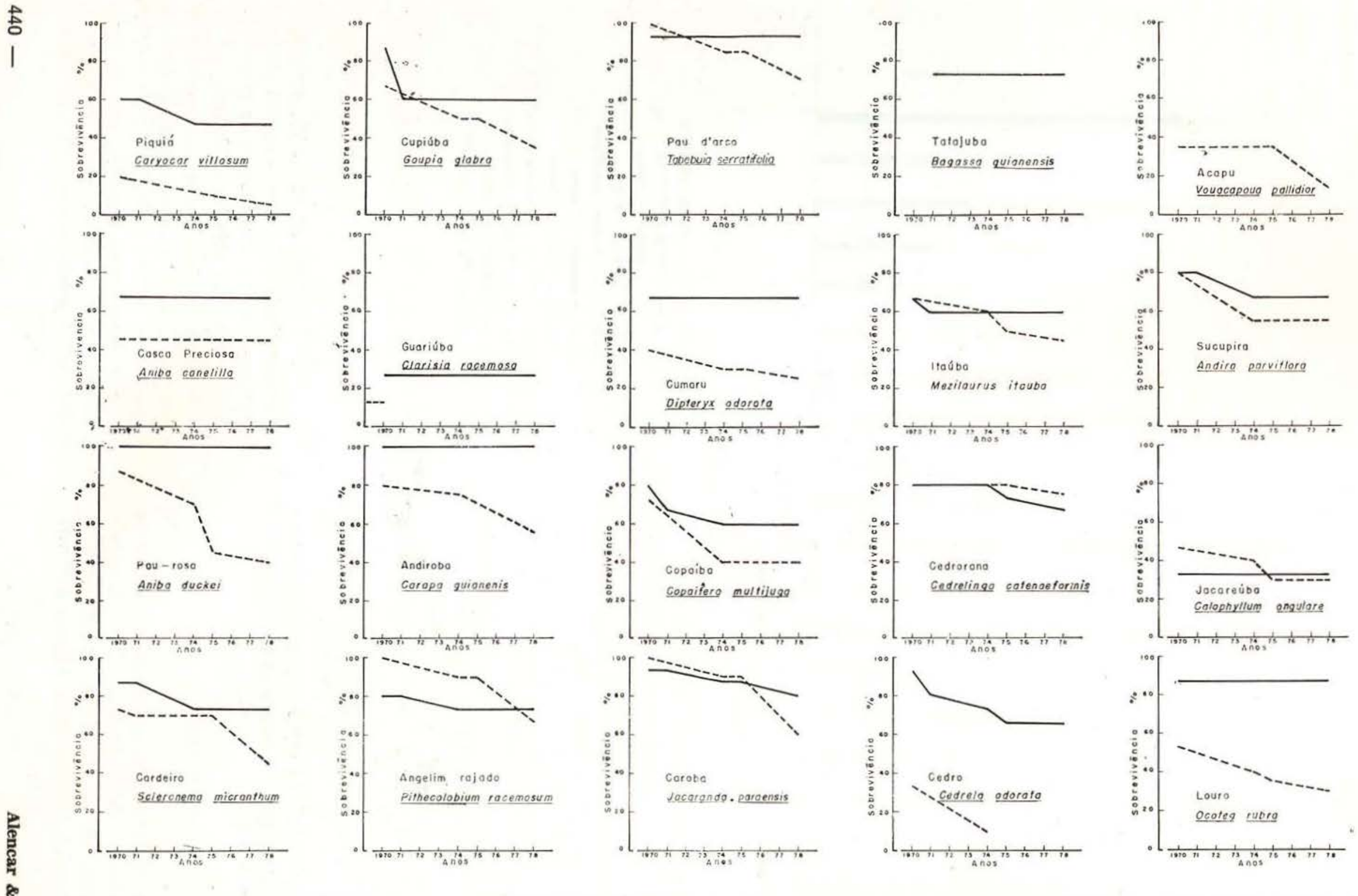

Fig. 3 - Sobrevivência de espécies florestais plantadas sob a sombra de floresta primária não explorada (-..-) e em plena abertura (-). 


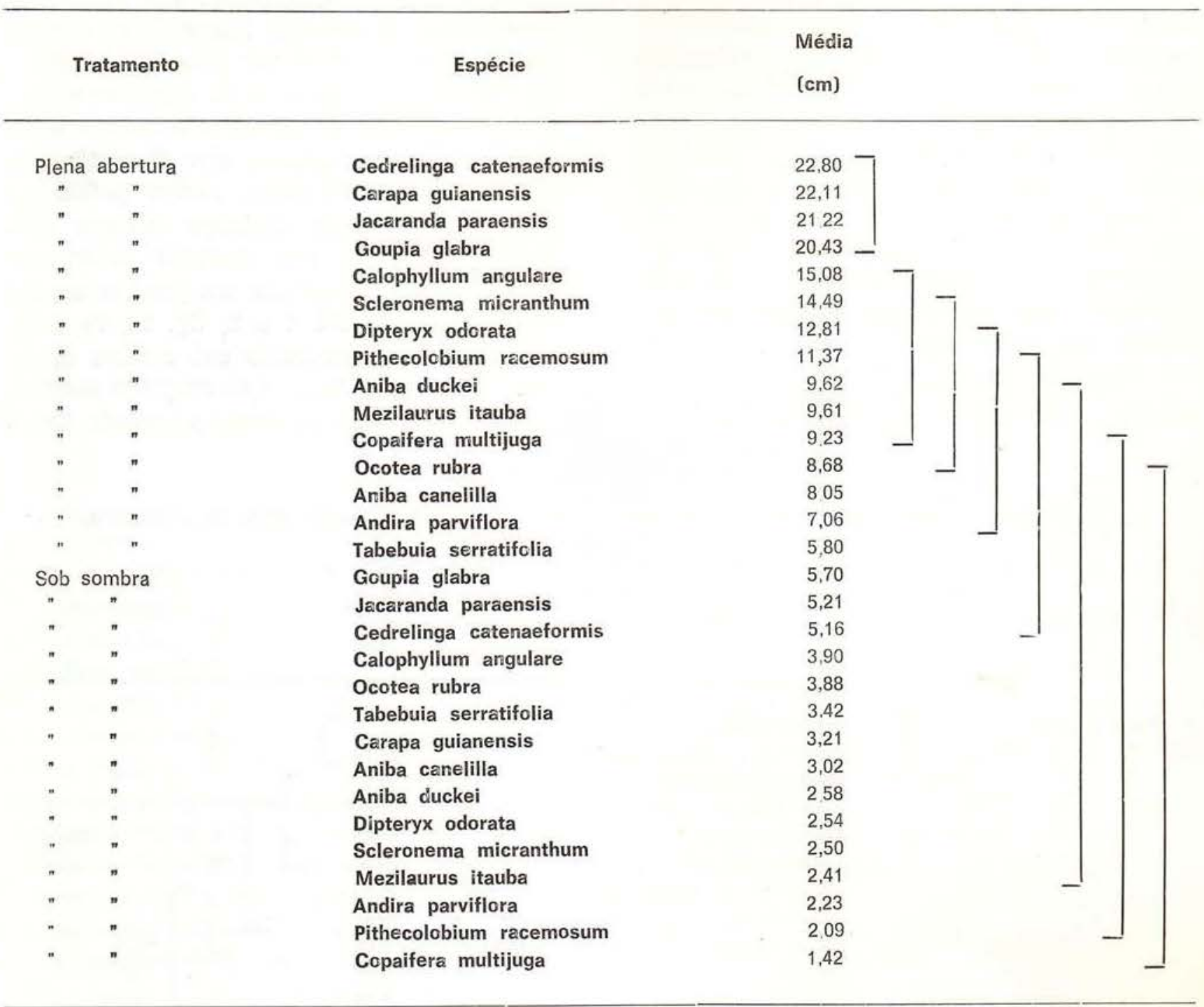

fator físico é de maior interesse ao ecólogo, do que a luz; porque ela é a fonte de energia para toda a vida, fator limitante e regulador da atividade diária e estacional de organismos vegetais e animais. Por isto ser verdade, o experimento agora exposto teve como objetivo maior verificar a importância do fator luz no desenvolvimento de espécies florestais, antes de pensar na determinação ou maximização de incrementos. A luz é, de fato, o fator fundamental no crescimento de árvores em zona tropical, principalmente quando se deseja realizar uma silvicultura com espécies mistas e sob sombra. Verificamos, pelos resultados da sobrevivência das espécies plantadas sob sombra da floresta primária não explorada comercialmente que apenas 4 espécies dentre as testadas (Cedrelinga catenaeformis, Tabebuia serratifolia, Pithecolobium racemosum e Jacaranda paraensis), apresentaram uma sobrevivência entre $60-79 \%$, enquanto em plena abertura 11 espécies alcançaram essa porcentagem, sendo que 5 espécies (Aniba duckei, Carapa guianensis, Tabebuia serratifolia, Ocotea rubra e Jacaranda paraensis) atingiram entre 80 a $100 \%$ de sobrevivência (Ver fig. 3).

Isto vem reforçar e confirmar a tese de que a luz, funcionando como um "freio", é um fator regulador da sobrevivência e limitante do cres. cimento dos vegetais. Convém ainda salientar. 
ressaltando aqui o conceito da "lei do mínimo" de Leibig, de que, tornando-se a taxa de luz tão baixa, ela poderá limitar o crescimento mesmo na presença de doses elevadas de fertilizantes, água ou outro fator. Araujo (1970), ao relatar resultados qualitativos deste experimento, afirmava que: concorda-se, segundo principios gerais, que a competição é mais ou menos restrita entre os individuos de uma comunidade, dependendo de suas necessidades. E por conseguinte, os elementos de competição são: primeiro a luta por espaço, segundo por luz e terceiro por nutrientes.

Conforme expusemos na introdução, se se deseja o desenvolvimento de uma silvicultura que funcione em harmonia com o ecossistema florestal amazônico, devemos dar atenção ao modo como as espécies florestais se comportam quando plantadas em diferentes níveis de luz. Verificamos entre as 21 espécies estudadas que maiores incrementos em altura e diâmetro podem ser obtidos com Goupia glabra, Cedrelinga catenaeformis, Carapa guianensis, Jacaranda paraensis, Dipteryx odorata, Calophyllum angulare, Pithecolobium racemosum e Scleronema micranthum em plantios em plena abertura (Quadro 1 e 2, fig. 1a, 1b e 2). Vimos que o crescimento sob sombra de floresta primária foi inferior ao em plena abertura, mas Goupia glabra, Jacaranda paraensis, Cedre-

QUADRO 2 - Média das alturas de 10 observações em ordem decrescente. Teste de Tukey (5\%)

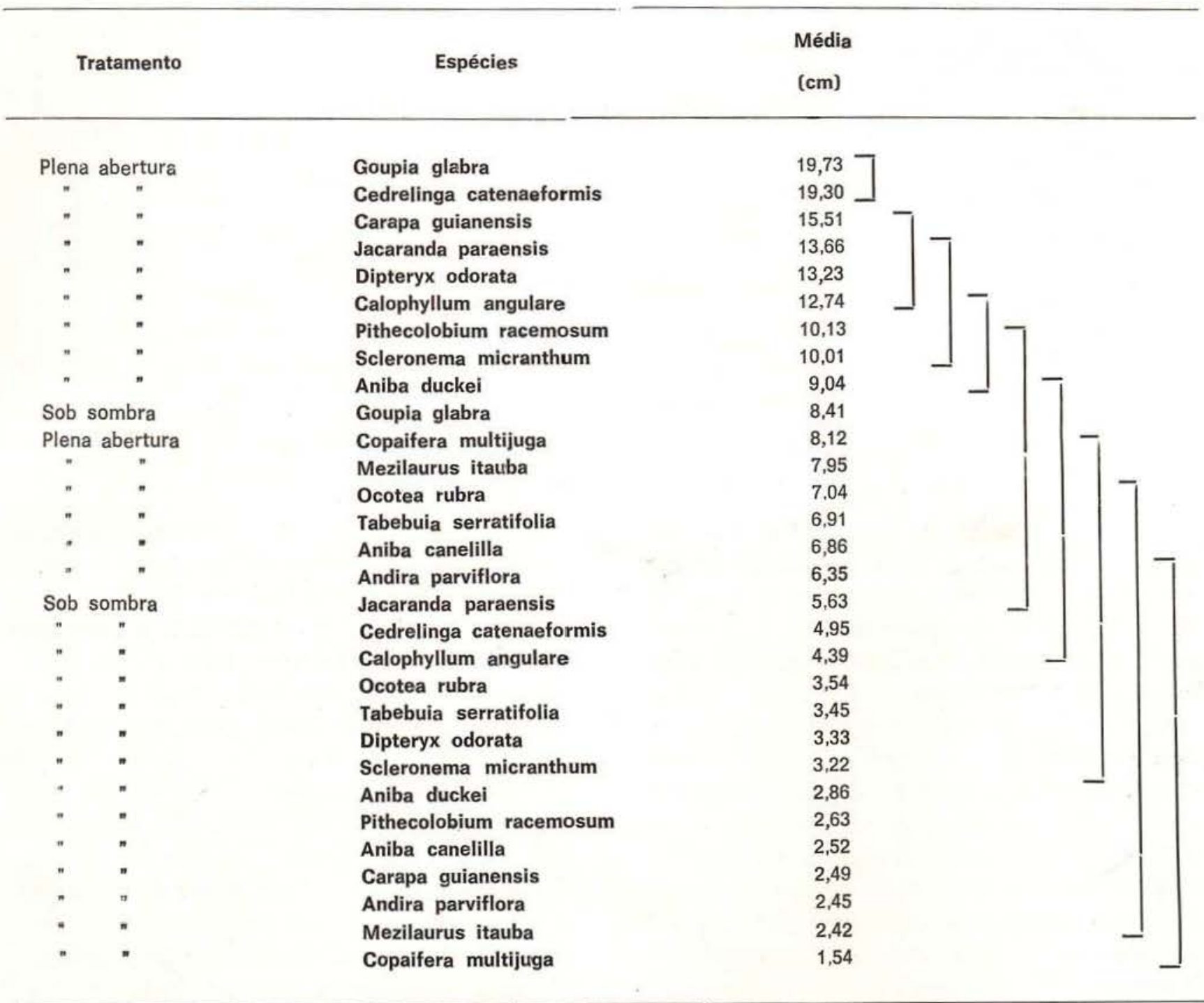


linga catenaeformis, Calophyllum angulare, Ocotea rubra, Tabebuia serratifolia, podem ser plantados sob sombra. Convém, ainda, chamar a atenção para o fato de que se tratou de dar um grau de sombra em floresta não explorada comercialmente, sem a realização de qualquer desbaste ou adubação química, porque se desejou ensaiar valores extremos. No caso de floresta já devidamente explorada e desde que o número de árvores remanescentes seja tec. nicamente conduzido, seguramente, ter-se-ão maiores crescimentos tanto em altura como em diâmetro para estas espécies com este trata. mento. Alencar et al. (s/d) sugerem o plantio nestas condições para Aniba duckei, uma vez que teve alta taxa de sobrevivência naquele experimento.

Concluindo, recomenda-se que nos futuros reflorestamentos em implantação na Amazônia, se dê preferência para plantios de Goupia glabra, Cedrelinga catenaeformis, Carapa guianen. sis, Jacaranda paraensis, Dipteryx odorata, Ca lophyllum angulare, Ocotea rubra, Pithecolo. bium racemosum, Scleronema micranthum e Aniba duckei, enquanto não se conheçam es. pécies mais promissoras com o desenvolvi. mento natural da ciência florestal nesta região. Para estas espécies, sugerimos que se façam estudos de procedências de sementes, espaça mentos e ensaios com aplicação de fertilizantes, ao mesmo tempo em que se deveria ini ciar o melhoramento destas essências.

\section{Agradecimentos}

Os autores agradecem ao Prof. Hugo Menezes dos Santos por sua valiosa colaboração na análise estatística dos dados; ao Sr. Joaquim Menezes Ferreira pela listagem e preparação de cartões para computação e a Eliana Minelli de Oliveira pela datilografia do texto. Agradecem ainda ao Dr. Guido Ranzani e Dr. William Rodrigues pela correção do texto.

\section{SUMMARY}

The authors compare the growth in diameter and height and the survival of 21 amazonian forest species, of economic value, planted under two hight conditions: one under the shade of undisturbed high forest and the other in full light.

They verify that the growth in height and diameter and the percentage survival, in full light, was superior to that under shade for almost all the species tested, showing that light is an important, fundamental factor as much as a limiting factor to growth as a regulator of survival of forest species. The species which presented the better appearance with the greater heights and diameters in full light were Goupia glabra, Cedrelinga catenaeformis, Bagassa guianensis, Carapa guianensis, Jacaranda paraensis, Dipteryx odorata, Calophyllum angulare, Pithecolobium racemosum and Scleronema micranthum. Under the shade of undisturbed high forest the better species were Goupia glabra, Jacaranda paraensis, Cedrelinga cateaneformis, Calophyllum angulare, Ocotea rubra e Tabebuia serratifolia. The authors recommend these species for plantations in the Amazon, calling attention to the fact that the knowledge of intrinsic ecological characteristics of each species is essential for silvicultural success in this region.

\section{BIBLIOGRAFIA}

Alencar, J.C. \& Fernandes, N.P.

1978 - Desenvolvimento de árvores nativas em ensaios de espécies. 1. Pau-rosa (Aniba duckei Kostermans). Acta Amazonica, 8(4): 523-541.

Alencar, J.C.: Fernandes, N.P. \& Loureiro, A.A.

$\mathrm{s} / \mathrm{d}$ - Desenvolvimento de árvores nativas em ensaios de espécies. 2. Jacareúba (Calophyllum angulare A.C. Smith) Acta Amazonica, (Entregue para publicação).

Araujo, V.C.

1970 - The factor light as a basic element in tree growth in the Amazonian forest. In: Symposium Proceedings on environment in Amazonia, Manaus, Part I: 67-77.

Falesi, I.C.; Cruz, E. De S.; Pereira, F.B. \& Lopes, E.C.

1969 - Os solos da área Manaus-ltacoatiara. IPEAN, Série Estudos e Ensaios, 1.

ODUM, E.P.

1977 - Ecologia; tradução de Hell, K.G. - 3.a Edição. Livraria Pioneira Editora. São Paulo.

PEdRoso, L.M.

1969 - Informações sobre o atual comportamento de espécies exóticas na região do baixo Amazonas. SUDAM 5(1/4) : 23-32. 
Pedroso, L.M.; Pereira, A.P.; Leite, P.F. \& Silva, C. DA

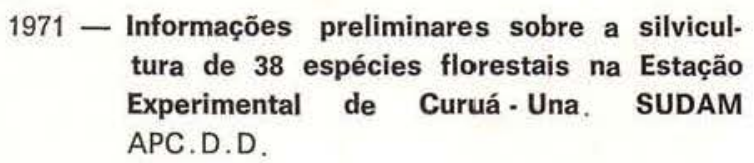

1971 - Informações preliminares sobre a silvicultura de 38 espécies florestais na Estação Experimental de Curuá-Una. SUDAM APC.D.D.

Volpato, E.; Schimidt, P.B. \& Araujo, V.C.

1972 - Carapa guianensis Aubl. (Andiroba). Estudos comparativos de tratamentos silviculturais. Acta Amazonica, 2(3) : 7581 .
1973 - Situação dos plantios experimentais na Reserva Ducke. Acta Amazonica, 3(1) : 71-82.

1979 - Pesquisas e informações sobre espécies florestais da Amazônia. SUDAM - Depto. Recursos Naturais e Centro de Tecnologia da Madeira. 111 p. il.

(Aceito para publicação em $20 / 03 / 80$ ) 